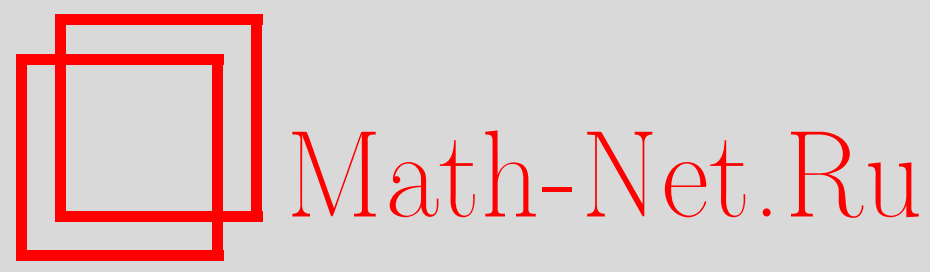

В. Н. Беляев, В. К. Булитко, $m$-сводимость с верхними и нижними границами для сводящих функций, Матем. заметки, 2001, том 70, выпуск 1, 12-21

DOI: https://doi.org/10.4213/mzm713

Использование Общероссийского математического портала Math-Net.Ru подразумевает, что вы прочитали и согласны с пользовательским соглашением http://www . mathnet.ru/rus/agreement

Параметры загрузки:

IP: 3.80 .181 .102

26 апреля 2023 г., $16: 31: 57$

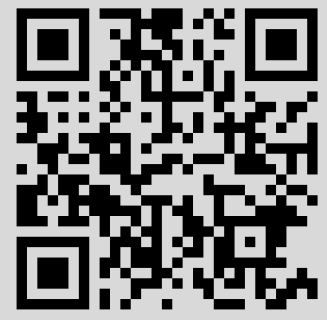




\title{
m-СВОДИМОСТЬ С ВЕРХНИМИ И НИЖНИМИ ГРАНИЦАМИ ДЛЯ СВОДЯщИХ ФУНКцИй
}

\author{
В.Н. Беляев, В. К. Булитко
}

Изучаются такие пары $\left(\mathfrak{T}^{1}, \mathfrak{T}^{0}\right)$ классов неубывающих тотальных одноместных арифметических функций, которые определяют рефлексивные и транзитивные бинарные отношения $\left\{(A, B) \mid A, B \subseteq N \&(\exists\right.$ о.p.ф.. $\left.h)\left(\exists f_{1} \in \mathfrak{T}^{0}\right)\left[A \leqslant{ }_{m}^{h} B \& f_{0} \unlhd h \unlhd f_{1}\right]\right\}$. (Здесь $k \unlhd l$ означает, что функция $l$ почти всюду мажорирует функцию $k$.) Установлены критерии рефлексивности и транзитивности таких отношений. Получены свидетельства высокой разветвленности возникающей системы ограниченных сводимостей $m$-типа. Построены примеры таких сводимостей, существенно отличающиеся от стандартной $m$-сводимости в отношении структуры порождаемых ими систем степеней неразрешимости и в вопросе полноты множеств.

Библиография: 4 названия.

$m$-сводимость является одной из основных сводимостей в теории рекурсии и достаточно хорошо изучена. Однако в прикладных вопросах часто она является все же слишком идеализированной из-за ничем, кроме рекурсивности сводящей функции, не ограниченного доступа к оракулу. Мы видим, что в теории сложности прикладных комбинаторных задач центральное место занимают ограничения вычислительной сложности сводящей функции. Целью настоящей заметки является рассмотрение последствий для $m$-сводимости, наступающих из-за ограничения доступа к оракулу в $m$-редукциях. Эти ограничения играют роль некоторой “дескриптивной сложности” $m$-редукций. Аналогичные ограничения (но только сверху) изучались ранее в [1], [2] для тьюринговых сводимостей.

Основные понятия и обозначения определены в [3]. Напомним, что сокращения р.м., р.п.м., о.р.ф. обозначают соответственно “рекурсивное(ые) множество(а)", "рекурсивно перечислимое множество(а)", "общерекурсивная(ые) функция (ии)".

Будем говорить, что $f \unlhd g$, если $\exists x_{0} \forall y\left[y>x_{0} \Longrightarrow f(y)<g(y)\right]$; функцию $g$ будем назьвать мажсорантой п.в. (почти всюду) функции $f$, а $f$-минорантой n.в. для $g$.

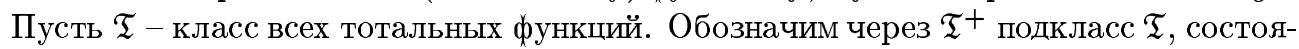
щий из всех неубьвающих функций, и пусть $\mathfrak{R}^{i} \subseteq \mathfrak{T}^{+}, i=0,1$. С каждой тотальной функцией $f$ можно связать две функции

$$
e_{f}^{1}(x)=\max \{f(y) \mid y \leqslant x\}, \quad e_{f}^{0}(x)=\min \{f(y) \mid x \leqslant y\} .
$$

ЛЕмма 1. Если $f$ о.р.ф., то $e_{f}^{1}$ неубьвающая о.р.ф., а $e_{f}^{0}$ предельно рекурсивная неубывающая функиия. 
ДоказАтЕльство. Рассмотрим второепредложение. Справедливо, очевидно, представление $e_{f}^{0}(x)=\lim _{t \rightarrow \infty} h(x, t)$, если положить $h(x, t) \rightleftharpoons \min \left\{f\left(x^{\prime}\right) \mid x \leqslant x^{\prime} \leqslant t\right\}$.

Введем основное определение:

$$
\begin{aligned}
\leqslant_{m}\left[\begin{array}{l}
\mathfrak{R}^{1} \\
\mathfrak{R}^{0}
\end{array}\right] \rightleftharpoons & \left\{(A, B) \in 2^{\mathbb{N}} \times 2^{\mathbb{N}} \mid\right. \\
& \left.\exists \text { o.p.фp. } f \exists h^{1} \in \mathfrak{R}^{1} \exists h^{0} \in \mathfrak{R}^{0}\left[A \leqslant{ }_{m}^{f} B \& h^{0} \unlhd e_{f}^{0} \& e_{f}^{1} \unlhd h^{1}\right]\right\} .
\end{aligned}
$$

Очевидно, $\leqslant_{m}\left[\begin{array}{l}\mathfrak{R}^{1} \\ \mathfrak{R}^{0}\end{array}\right] \subseteq \leqslant_{m}$. Будем называть отношение $\leqslant_{m}\left[\begin{array}{l}\mathfrak{R}^{1} \\ \mathfrak{R}^{0}\end{array}\right]$ ограниченной $m$-сводимостью (или просто сводимостью), если это отношение рефлексивно и транзитивно.

Наложение ограничений снизу на доступ к оракулу мотивируется тем, что редукции (сведе́ния) с ограничениями такого типа не только позволяют оценить сложность сводимого множества по сложности оракула, но и в определенной мере переносят строение сводимого множества на оракул. Например, ясно, что невозможна редукция рекурсивного бесконечного множества к иммунному, если только нижняя граница этого сведения есть бесконечнозначная неубывающая функция. Эти связи демонстрируют также некоторые из результатов настоящей заметки.

Первой целью заметки является установление условий рефлексивности и транзитивности отношений рассматриваемого в работе типа. Затем будут указаны некоторые структурные свойства семейства найденных сводимостей, а также определенные особенности, не имеюшие места для классической $m$-сводимости.

Если $\mathfrak{A}, \mathfrak{B} \subseteq \mathfrak{T}^{+}$и $(\forall f \in \mathfrak{A})(\exists g \in \mathfrak{B})[f \unlhd g]$, то пишем $\mathfrak{A} \unlhd^{1} \mathfrak{B}$, а если $(\forall g \in \mathfrak{B})$ $(\exists f \in \mathfrak{A})[f \unlhd g]$, то пишем $\mathfrak{A} \unlhd^{0} \mathfrak{B}$.

Лемма 2. 1) Пусть даны произвольные $\mathfrak{B}^{i} \subseteq \mathfrak{T}^{+}, i=0,1$, и такие $\mathfrak{A}^{i} \subseteq \mathfrak{T}^{+}$, $i=0,1$, ито $\mathfrak{B}^{1} \unlhd^{1} \mathfrak{A}^{1} \& \mathfrak{A}^{0} \unlhd^{0} \mathfrak{B}^{0}$. Tогдa $\leqslant_{m}\left[\begin{array}{c}\mathfrak{B}^{1} \\ \mathfrak{B}^{0}\end{array}\right] \subseteq \leqslant_{m}\left[\begin{array}{l}\mathfrak{A}^{1} \\ \mathfrak{A}^{0}\end{array}\right]$.

2) Всякое отношение вида $\leqslant_{m}\left[\mathfrak{R}^{1}\right]$ можсно задать счетными подклассами $\mathfrak{T}^{+}$.

ДокАЗАтЕльство. 1) Действительно, пусть $C \leqslant_{m}\left[\begin{array}{l}\mathfrak{B}^{1} \\ \mathfrak{B}^{0}\end{array}\right] D$. Это по определению значит, что $\left(\exists h^{1} \in \mathfrak{B}^{1}\right)\left(\exists h^{0} \in \mathfrak{B}^{0}\right)\left[C \leqslant{ }_{m}^{f} D \& h^{0} \unlhd e_{f}^{0} \& h^{1} \unrhd e_{f}^{1}\right]$. Из условия леммы следует, что $\left(\exists g^{0} \in \mathfrak{A}^{0}\right)\left(\exists g^{1} \in \mathfrak{A}^{1}\right)\left[g^{0} \unlhd h^{0} \& g^{1} \unrhd h^{1}\right]$.

Далее, из транзитивности отношения $\unlhd$ следует, что $\left[C \leqslant{ }_{m}^{f} D \&\left(g^{0} \unlhd e_{f}^{0}\right) \&\left(e_{f}^{1} \unlhd g^{1}\right)\right]$, т.е. $C \leqslant_{m}\left[\begin{array}{l}\mathfrak{A}^{1} \\ \mathfrak{A}^{0}\end{array}\right] D$.

Второе предложение леммы следует из того факта, что множество $\left\{e_{f}^{i} \mid f\right.$ о.р.ф., $i=\overline{0,1}\}$ очевидно счетно.

Далее, если не указано обратное, будем в отношениях $\leqslant m\left[\begin{array}{l}\mathfrak{R}^{1} \\ \mathfrak{R}^{0}\end{array}\right]$ считать $\mathfrak{R}^{0}, \mathfrak{R}^{1}$ счетными.

СлЕДСТВИЕ 1. Существуют такие $h^{\star}, l^{\star} \in \mathfrak{T}^{+}$, что $\leqslant_{m}=\leqslant_{m}\left[\begin{array}{l}h^{\star} \\ l^{\star}\end{array}\right]$.

ДокАЗАТЕЛЬСтво. В качестве $l^{\star}$ можно взять $\lambda x[0 \cdot x]$.

Что касается $h^{\star}$, то подходит любая неубываюшая тотальная мажоранта п.в. класса всех о.p.ф. Если положить $h(x, t)=\max \left\{\varphi_{i}(y) \mid i \leqslant x, y \leqslant x, \varphi_{i}(y) \downarrow\right.$ за не более чем $t$ шагов $\}$, то ясно, что $h^{\star}(x)=\lim _{t \rightarrow \infty} h(x, t)$ определена для всех $x \in \mathbb{N}$ и мажорирует п.в. все о.р.ф.

$$
\text { Положим } \mathfrak{T}^{+, \infty} \rightleftharpoons\left\{f\left|f \in \mathfrak{T}^{+} \&\right| V \text { al } f \mid=\infty\right\} .
$$


Следующая лемма показьвает, что характеризация классов нижних границ, определяюших сводимости, является более сложной задачей по сравнению с такой же проблемой для классов верхних границ. Далее будет еще несколько иных подтверждений сказанного.

Лемма 3. Для любой рекурсивной функиии $f$ с условием $\exists y\left[\left|f^{-1}(y)\right|=\infty\right]$ мож-

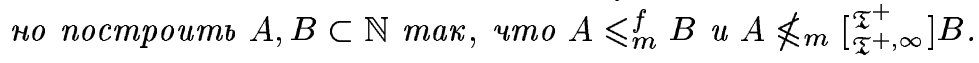

ДокАЗАТЕЛЬСТво. Выберем в качестве $B$ иммунное множество, включающее некоторое $y \in V$ al $f$ с бесконечным прообразом $H$. Положим $A=f^{-1}(B)$. Ясно, что $H \subseteq A$.

$\overline{\Pi р е д п о л о ж и м ~ т е п е р ь, ~ ч т о ~ д л я ~ н е к о т о р о и ̆ ~ о . p . ф . ~} g$ верно $A \leqslant{ }_{m}^{g}\left[\left[_{\mathfrak{T}}^{+}+, \infty\right] B\right.$. Тогда $g(H) \subseteq B$ и в силу иммунности $B$ множество $g(H)$, будучи рекурсивно перечислимьп, должно быть конечным. Однако тогда тотальная функция $g$ имела бы конечньй образ на некотором бесконечном множестве. Это невозможно, очевидно, совместить с наличием для $g$ неубывающей тотальной нижней гранищы п.в., у которой множество значений бесконечно.

ЛЕмма 4. Пусть $\mathfrak{R} \subseteq \mathfrak{T}^{+}$.

(а) Если для о.р.ф. $f$ верно $\mid V$ al $f \mid=\infty,\{f\} \mathbb{\unlhd}^{1} \mathfrak{R}$, то существуют такие $A, B \subseteq \mathbb{N}$, что $A \leqslant_{m}^{f} B \&: A \nless_{m}\left[{ }_{\mathfrak{T}+}^{\Re}\right] B$.

(б) Если для о.р.ф. $f$ верно, что $e_{f}^{0} \in \mathfrak{T}^{+, \infty}, \mathfrak{R} \not^{0}\left\{e_{f}^{0}\right\}$, то существуют такие $A, B \subseteq \mathbb{N}$, что $A \leqslant{ }_{m}^{f} B \& A \nless_{m}\left[\stackrel{\mathfrak{T}}{\mathfrak{R}}^{+}\right] B$.

ДоказАтЕльство. (а) Пусть $f_{0}, f_{1}, \ldots$ список тех о.р.ф., у которых $e_{f_{0}}^{1}, e_{f_{1}}^{1}, \ldots$ имеют мажоранты п.в. в $\mathfrak{R}^{1}$. Пусть для $g_{n} \in \mathfrak{R}^{1}$ верно $g_{n} \unrhd e_{f_{n}}^{1}, n \in \mathbb{N}$. Так как $\{f\} \unlhd^{1} \mathfrak{R}^{1}$, то $\forall n \in \mathbb{N}\left[f \notin g_{n}\right]$. Для каждого $n$ введем в рассмотрение последовательность чисел $x_{0}^{n}<x_{1}^{n}<x_{2}^{n}<\cdots$, выполняющую условия

$$
e_{f_{n}}^{1}\left(x_{k}^{n}\right) \leqslant g_{n}\left(x_{k}^{n}\right)<f\left(x_{k}^{n}\right)<f\left(x_{k+1}^{n}\right), \quad k \in \mathbb{N}
$$

(последнее неравенство достижимо из-за $\mid V$ al $f \mid=\infty$ ), и

$$
\forall y\left[e_{f_{n}}^{1}\left(y+x_{0}^{n}\right) \leqslant g_{n}\left(y+x_{0}^{n}\right)\right] .
$$

Построим $\sigma: \mathbb{N} \rightarrow \mathbb{N}$ так, что

$$
\forall n\left[f\left(x_{\sigma(n)}^{n}\right)>g_{n}\left(x_{\sigma(n)}^{n}\right) \& x_{\sigma(n)}^{n}<x_{\sigma(n+1)}^{n+1}\right] .
$$

Положим $B=f(A), A \subset\left\{x_{\sigma(n)}^{n} \mid n \in \mathbb{N}\right\}$. Пусть далее $\mathscr{C}_{A}$ определена уже на $\left[0, x_{\sigma(n-1)}^{n-1}\right]$. Определим

$$
\mathscr{C}_{A}\left(x_{\sigma(n)}^{n}\right)=1 \Longleftrightarrow f_{n}\left(x_{\sigma(n)}^{n}\right) \notin B .
$$

Это возможно, так как $f\left(x_{\sigma(n)}^{n}\right)>e_{f_{n}}^{1}\left(x_{\sigma(n)}^{n}\right)$ и определение положения $x_{\sigma(n)}^{n}$ относительно $A$ не отражается на принадлежности $f_{n}\left(x_{\sigma(n)}^{n}\right)$ к $B$. Ясно, что $A \leqslant f$, $B$, но $A{\underset{m}{m}}_{f_{n}} B$, так как иначе на $x_{\sigma(n)}^{n}$ было бы противоречие.

(б) Пусть $\left(r_{i}\right)_{i \in \mathbb{N}}$ список о.р.ф., удовлетворяюших условие $\mathfrak{R} \unlhd^{0}\left\{e_{r_{i}}^{0} \mid i \in \mathbb{N}\right\}$. Если этот список пуст, то доказывать нечего. Из условия следует $\forall i\left[e_{r_{i}}^{0} \not e_{f}^{0}\right]$. А значит, $\forall i$ 
$\left[r_{i} \not f\right]$. Поэтому для всякого натурального $i$ существует бесконечно много чисел $z_{k}^{i}$, $k=0,1, \ldots$ таких, что $\forall k\left[r_{i}\left(z_{k}^{i}\right)>f\left(z_{k}^{i}\right)\right]$.

Из последовательности $\left(z_{k}^{i}\right)_{k \in \mathbb{N}}$ можно выбрать подпоследовательность $\left(y_{s}^{i}\right)_{s \in \mathbb{N}}$ так, чтобы $\forall s\left[r_{i}\left(y_{s}^{i}\right)>f\left(y_{s}^{i}\right)\right] \& \forall s\left[r_{i}\left(y_{s}^{i}\right)<f\left(y_{s+1}^{i}\right)\right]$. То есть вьполнена следуюшая система неравенств:

$$
\cdots<f\left(y_{s}^{i}\right)<r_{i}\left(y_{s}^{i}\right)<f\left(y_{s+1}^{i}\right)<r_{i}\left(y_{s+1}^{i}\right)<\cdots .
$$

Теперь остается только с помощњю подходящей функции $\sigma: \mathbb{N} \rightarrow \mathbb{N}$ построить диагональную последовательность чисел $y_{\sigma(i)}^{i}$ так, чтобы для любого $i$ вьполнялись неравенства

$$
f\left(y_{\sigma(i)}^{i}\right)<r_{i}\left(y_{\sigma(i)}^{i}\right)<f\left(y_{\sigma(i+1)}^{i+1}\right)<r_{i+1}\left(y_{\sigma(i+1)}^{i+1}\right) .
$$

Построим множество $B$ путем выбора для каждого $i \in \mathbb{N}$ одного и только одного элемента из пары чисел $\left\{f\left(y_{\sigma(i)}^{i}\right), r_{i}\left(y_{\sigma(i)}^{i}\right)\right\}$. А затем определим множество $A$ условием $A=f^{-1}(B)$. Это обеспечивает $A \leqslant{ }_{m}^{f} B$. Нетрудно убедиться, однако, что $\forall i\left[A \nless_{m}^{r_{i}} B\right]$. Поэтому $A \leqslant{ }_{m}^{f} B \&: A \Varangle_{m}\left[\mathfrak{\mathfrak { R }}_{\mathfrak{R}}^{+}\right] B$.

Теорема 1. Для любих $\mathfrak{R}^{1}, \mathfrak{R}^{0} \in \mathfrak{T}^{+}$отношение $\leqslant_{m}\left[\begin{array}{l}\mathfrak{R}^{1} \\ \mathfrak{R}^{0}\end{array}\right]$ рефлексивно тогда и только тогда, когда $\mathfrak{R}^{0} \unlhd^{0}\{\mathscr{I}\} \unlhd \mathfrak{R}^{1}$.

ДокАЗАТЕЛЬСтво. Достаточность очевидна, ибо $A \leqslant m\left[\begin{array}{l}\mathfrak{R}^{1} \\ \mathfrak{R}^{0}\end{array}\right] A$ со сводящей функцией $\mathscr{I}$.

Необходимость. Если для $\mathscr{I}$ нет миноранты п.в. в $\mathfrak{R}^{0}$, то по лемме 4 найдутся такие $A, B \in \mathbb{N}$, что $A \leqslant_{m}^{\mathscr{I}} B$, т.е. $A=B$, но $A \Varangle_{m}\left[\begin{array}{l}\mathfrak{T}_{\mathfrak{R}}^{+} \\ \mathfrak{R}^{0}\end{array}\right] A$. То же можно сказать и в случае, когда для $\mathscr{I}$ нет мажоранты п.в. в $\mathfrak{R}^{1}$.

На основе леммы 4 можно доказать следуюший результат.

Теорема 2. Отношение $\leqslant_{m}\left[\begin{array}{l}\mathfrak{R}^{1} \\ \mathfrak{R}^{0}\end{array}\right]$ транзитивно тогда и только тогда, когда

$$
\begin{aligned}
(\forall \text { o.p. } . \text {. f }, g)\left[\left\{e_{f}^{1}, e_{g}^{1}\right\} \unlhd^{1} \mathfrak{R}^{1} \& \mathfrak{R}^{0} \unlhd^{0}\left\{e_{f}^{0}, e_{g}^{0}\right\}\right. \\
\quad \Longrightarrow\left\{e_{f}^{1}, e_{g}^{1} \in \mathfrak{T}^{+, \infty} \Longrightarrow\left(\exists H^{1} \in \mathfrak{R}^{1}\right)\left[e_{f \circ g}^{1} \unlhd H^{1}\right]\right\} \&\left\{e_{f}^{0}, e_{g}^{0} \in \mathfrak{T}^{+, \infty}\right. \\
\left.\left.\quad \Longrightarrow\left(\exists H^{0} \in \mathfrak{R}^{0}\right)\left[H^{0} \unlhd e_{f \circ g}^{0}\right]\right\}\right] \&\left\{( \exists d \in \mathbb { N } ) \left[\mathfrak{R}^{0} \unlhd^{0}\{d\} \unlhd^{1} \mathfrak{R}^{1}\right.\right. \\
\left.\left.\quad \Longrightarrow(\forall d \in \mathbb{N})\left[\mathfrak{R}^{0} \unlhd^{0}\{d\} \unlhd^{1} \mathfrak{R}^{1}\right]\right]\right\} .
\end{aligned}
$$

ДокАЗАТЕЛЬСтво. Достаточность. Правая часть эквивалентности есть конъюнкция двух условий, второе из которых будем называть условием констант. Следствие первого условия правой части эквивалентности есть конъюнкция двух условий-импликаций, заключенных в фигурные скобки, которые обозначим для краткости $\{1 \ldots\}_{1}$ и $\{2 \ldots\}_{2}$ соответственно.

Предположим дано $A \leqslant{ }_{m}^{g}\left[\begin{array}{l}\mathfrak{R}^{1} \\ \mathfrak{R}^{0}\end{array}\right] B \leqslant{ }_{m}^{f}\left[\begin{array}{l}\mathfrak{R}^{1} \\ \mathfrak{R}^{0}\end{array}\right] C$.

Если $e_{f}^{0}, e_{g}^{0} \in \mathfrak{T}^{+, \infty}$, то условия в скоб̆ках $\{1 \ldots\}_{1}$ и $\{2 \ldots\}_{2}$ выполнены для данных о.p.ф. $f, g$, т.е. посылки импликаций истинны. Тогда, очевидно, из $A \leqslant{ }_{m}^{g} B \& B \leqslant{ }_{m}^{f} C$ следует $A \leqslant{ }_{m}^{f \circ g}\left[\mathfrak{R}_{0}^{\Re^{1}}\right] C$, соответствующее условию транзитивности потому, что в данном случае поведение функций (и множеств) на начальных сегментах натурального ряда не имеет значения.

Если неверно, что $e_{f}^{0}, e_{g}^{0} \in \mathfrak{T}^{+, \infty}$, то по условию констант из $\left\{{ }_{3} \ldots\right\}_{3}$ должно быть $\mathfrak{R}^{0} \unlhd^{0}\{0\}$. Фактически можно полагать $0 \in \mathfrak{R}^{0}$. В таком случае нет никаких проблем 
с нижними границами при построении сведения $A \leqslant m\left[\begin{array}{l}\mathfrak{R}^{1} \\ \mathfrak{R}^{0}\end{array}\right] C$. И если $e_{f}^{1}, e_{g}^{1} \in \mathfrak{T}^{+, \infty}$, то по условию $\{1 \ldots\}_{1}$ в качестве сводящей функции можно взять $f \circ g$.

Если, наконец, неверно $e_{f}^{1}, e_{g}^{1} \in \mathfrak{T}^{+, \infty}$, то пусть, например, $e_{g}^{1} \notin \mathfrak{T}^{+, \infty}$. Тогда по условию констант можно считать $0 \in \mathfrak{R}^{0}$ и $\mathfrak{R}^{1}$ мажорирует любую константу. Потому легко построить требуемое сведение рекурсивного множества $A$ к $C$.

Необходимость. Лемма 4 решает вопрос от противного в случае $e_{f}^{0}, e_{g}^{0} \in \mathfrak{T}^{+, \infty}$.

Предположим, отношение $\leqslant m\left[\mathfrak{R}_{\mathfrak{R}^{1}}^{1}\right]$ транзитивно, но условие констант не вьполнено. Это значит, что существует такое $d \in \mathbb{N}$, что $\mathfrak{R}^{0} \unlhd^{0}\{d\} \unlhd^{1} \mathfrak{R}^{1}$, но также существует и такое $b \in \mathbb{N}$, для которого неверно $\mathfrak{R}^{0} \unlhd^{0}\{b\} \unlhd^{1} \overline{\mathfrak{R}}^{1}$.

Рассмотрим сначала случай, когда в качестве $b$ подходит любое натуральное число, отличное от $d$ (т.е. $\exists ! d\left[\mathfrak{R}^{0} \unlhd^{0}\{d\} \unlhd^{1} \mathfrak{R}^{1}\right]$ ). Зафиксируем $b, b \neq d$, и построим множества $A, B, C$, а также цепочку сведений: $A \leqslant{ }_{m}^{g} B \leqslant_{m}^{f} C$ в качестве контрпримера к транзитивности $\leqslant m\left[\begin{array}{c}\mathfrak{R}^{1} \\ \mathfrak{R}^{0}\end{array}\right]$. Положим $A=\mathbb{N} \backslash\{b\}, B=\{d\}, C=\{b\}$. Далее

$$
g(x)=\left\{\begin{array}{l}
b, \text { если } x=b, \\
d \text { иначе, }
\end{array} \quad f(x)= \begin{cases}b, & \text { если } x=d, \\
d \text { иначе. }\end{cases}\right.
$$

Из построения следует, что если $A \leqslant m$, $C$, то $\left|q^{-1}(b)\right|=\infty$. Это означает невозможность $A \leqslant m\left[\begin{array}{l}\mathfrak{R}^{1} \\ \mathfrak{R}^{0}\end{array}\right] C$ в рассматриваемом случае.

Предположим теперь $d \neq b, d, b \in P$, где $P=\left\{a \mid \mathfrak{R}^{0} \unlhd^{0}\{a\} \unlhd^{1} \mathfrak{R}^{1}\right\}$. Покажем, что $a \in P$ для любого натурального $a$. Можно, очевидно, считать $a \notin\{b, d\}$. Определим множества $A=\{2 x \mid x \in \mathbb{N}\}, B=\{b\}, C=\{a\}$ и сводяшие функции

$$
g(x)=\left\{\begin{array}{l}
b, \text { если } x \in A, \\
d \text { иначе, }
\end{array} \quad f(x)=\left\{\begin{array}{l}
a, \text { если } x=b, \\
d \text { иначе. }
\end{array}\right.\right.
$$

Снова $A \leqslant \underset{m}{q} C$ влечет, что $q$ бесконечно много раз принимает значение $a$. Следовательно по транзитивности должно быть $a \in P$. Таким образом, условие констант вытекает из транзитивности, и теорема доказана.

Полученные результаты показывают, что для задания рассматриваемого типа сводимостей в качестве классов верхних границ (будем говорить короче - верхних классов) можно выбирать не более чем счетные системы неубываюших общерекурсивных функций, мажорирующих п.в. тождественную функцию $\mathscr{I}$, а в качестве классов нижних границ (нижних классов) - не более чем счетные системы предельно рекурсивных функций из $\mathfrak{T}^{+}$вида $e_{f}^{0}$, где $f-$ о.р.ф., минорирующих почти всюду $\mathscr{I}$. Такие классы будем назьвать приведенными.

Приведенные классы, задаюшие сводимости, будем назьвать транзитивными.

СлЕДСТВИЕ 2. Всякий приведенный верхний класс транзитивен тогда и только тогда, когда для всякой суперпозиции своих әлементов он включает маэсоранту п.в. А чтобы приведенный нижний класс был транзитивным, достаточно, чтобы для всякой суперпозичии своих әлементов он включал миноранту п.в.

ДокАЗАТЕЛЬСТво первого утверждения (критерия) непосредственно следует из теоремы 2.

Переходя к достаточному условию, заметим, что для любой тотальной функции $t$ верно $t \geqslant e_{t}^{0}$, а если $t \in \mathfrak{T}^{+}$, то $t=e_{t}^{0}$. Поэтому $e_{f}^{0} \circ e_{g}^{0} \leqslant f \circ g$. Теперь из $t \leqslant h$ следует 
$e_{t}^{0} \leqslant e_{h}^{0}$. Поэтому $e_{f}^{0} \circ e_{g}^{0} \leqslant e_{f \circ g}^{0}$ верно для любых тотальных $f, g$. Следовательно, если $e_{f}^{0} \circ e_{g}^{0}$ имеет миноранту, то это верно и для $e_{f \circ g}^{0}$.

Это следствие полезно для построения конкретных сводимостей рассматриваемого в работе типа.

Пусть дана система подмножеств $\mathfrak{s} \subseteq 2^{\mathbb{N}}$, замкнутая относительно объединения и включающая пустое множество. Будем говорить, что функции $f, g: \mathbb{N} \rightarrow \mathbb{N}$ совпадают с точностью до $\mathfrak{s}$ обозначая $f={ }_{\mathfrak{s}} g$, если $\{x \mid f(x) \neq g(x)\} \in \mathfrak{s}$. Ясно, что $=_{\mathfrak{s}}$ является отношением эквивалентности. В частности, $f \unlhd g \& g \unlhd f$ эквивалентно $f={ }_{\mathfrak{f}} g$, где $\mathfrak{f}$ обозначает класс всех конечных подмножеств $\mathbb{N}$.

Важную роль далее играют сингулярные (одноэлементные) классы функций, способные задавать сводимость. Сначала опишем некоторьй способ порождать такие классы.

Пусть $f, g \in \mathfrak{T}^{+}, f \geqslant g$. Определим $\widehat{f g}(0)=f(0)$ и

$$
\widehat{f g}(x+1)= \begin{cases}\widehat{f g}(x), & \widehat{f g}(x) \geqslant g(x+1), \\ f(x+1), & \widehat{f g}(x)<g(x+1) .\end{cases}
$$

Назовем $h$-гибридной функцию $g$, если она совпадает почти всюду с функцией $\widehat{h g}$ или с функцией $\widehat{g h}$.

Минимальной точкой одноместной функции $t: \mathbb{N} \rightarrow \mathbb{N}$ будем назьвать всякое такое значение ее аргумента $x$, что $(\forall y>x)[t(y)>t(x)]$. Обозначим через $M_{t}$ множество минимальных точек функции $t$.

Лемма 5. Пусть $\widehat{f g}$ определено. Тогда

1) $\widehat{f g} \in \mathfrak{T}^{+}$

2) $g \leqslant \widehat{f g} \leqslant f$

3) $е$ сли $g \unlhd \mathscr{I} \unlhd f$, mo $\widehat{f g} \circ \widehat{f g}=\mathfrak{f} \widehat{f g}$;

4) $\left(\forall h \in \mathfrak{T}^{+}\right)[h=\widehat{h h}]$;

5) для любых $h, g \in \mathfrak{T}^{+}$ecлu $h \unlhd g u g \circ g \unlhd h \circ g u л u g \unlhd h u h \circ g \unlhd g \circ g$, mo существует такая $h$-гибридная функиия $f$, ито $h \unlhd g \unlhd f$ или $f \unlhd g \unlhd h$ соответственно. Если еще $h={ }_{\mathfrak{f}} \mathscr{I}$, то g является $\mathscr{I}$-гибридной.

ДоказАтЕльство. 1) Если $\widehat{f g}(x) \geqslant g(x+1)$, то $\widehat{f g}(x+1) \geqslant \widehat{f g}(x)$. Иначе $\widehat{f g}(x+1)=$ $f(x+1)$. Но $f(x+1) \geqslant g(x+1)$, а $\widehat{f g}(x)<g(x+1)$. Поэтому снова $\widehat{f g}(x+1) \geqslant \widehat{f g}(x)$.

2 ) Для $x=0$ утверждение вытекает из условия $\widehat{f g}(0)=f(0)$. Пусть 2) доказано для $x \leqslant n$. Имеем $\widehat{f g}(n+1)=\widehat{f g}(n)$, если $\widehat{f g}(n) \geqslant g(n+1)$. Но $\widehat{f g}(n) \leqslant f(n)$ по индукции, а $f \in \mathfrak{T}^{+}$. Поэтому $g(n+1) \leqslant \widehat{f g}(n+1) \leqslant f(n+1)$. Если $\widehat{f g}(n)<g(n+1)$, то $\widehat{f g}(n+1)=f(n+1)$. Но по условию $f \geqslant g$.

3) Из определения следует, что $\widehat{f g}$ является ступенчатой неубывающей функцией. Рассмотрим отношение эквивалентности $x \equiv \widehat{f g} y \Longleftrightarrow \widehat{f g}(x)=\widehat{f g}(y)$. Непосредственно из определения вытекает, что каждый класс эквивалентности $\equiv \widehat{f g}$ является отрезком натурального ряда и, начиная с некоторого достаточно удаленного от начала, включает (ввиду условия $g \unlhd \mathscr{I} \unlhd f$ ) значение функции $\widehat{f g}$ на своих элементах. Это и означает требуемое равенство почти всюду суперпозиции $\widehat{f g} \circ \widehat{f g}$ самой функции $\widehat{f g}$.

4) Очевидно.

5) Рассмотрим один из указанных в условии случаев: $g \unlhd h \& h \circ g \unlhd g \circ g$. Тогда $h \circ g=_{\mathfrak{f}} g \circ g$ ввиду неубывания $g, h$. Следовательно, можно считать, что $h$ и $g$ совпадают 
почти всюду на $M_{g}$. Случай заключительной постоянности $g$ очевиден. Поэтому можно считать, что $\left|M_{g}\right|=\infty$. Пусть $\left\{x_{k} \mid k \in \mathbb{N}\right\}$ - пересчет $M_{g}$ в порядке возрастания элементов. Тогда $\mathbb{N}$ разбивается в сумму отрезков $\mathbb{N}_{k}=\left\{y \mid x_{k} \leqslant y<x_{k+1}\right\}, k \in \mathbb{N}$. Определим $f(x)=g\left(x_{k}\right), x \in \mathbb{N}_{k}, k \in \mathbb{N}$. Теперь утверждение очевидно.

Наконец, пусть дополнительно $h=_{\mathfrak{f}} \mathscr{I}$. Тогда сразу же получаем $g=_{\mathfrak{f}} g \circ g$. Рассмотрим произвольный достаточно большой (так чтобы вьполнялось равенство $g=g \circ g$ ) элемент $x$ из $M_{g}$. Имеем $x+1 \geqslant g(x+1)>g(x)$. Далее, если $x+1>g(x+1)$, то $g(x+1) \leqslant x$ и, значит, $g(x+1)>g(g(x+1))$. Это противоречие приводит к равенству $g=\mathscr{I}$ на всех достаточно больших числах вида $x+1, x \in M_{g}$. Поэтому $g$ является $\mathscr{I}$-гибридной.

ТЕОремА 3. Для любых классов $\mathfrak{R}^{1}, \mathfrak{R}^{0} \subseteq \mathfrak{T}^{+}$, удовлетворяюших условиям теорем 1 и 2, и любих $f, g \in \mathfrak{T}^{+}$таких, что $f \geqslant \mathscr{I} \geqslant g$, следующие бинарные отношения являются сводимостями

$$
\leqslant m\left[\begin{array}{l}
\widehat{f \mathscr{I}} \\
\mathfrak{R}^{0}
\end{array}\right], \quad \leqslant m\left[\frac{\mathfrak{R}^{1}}{\mathscr{I} g}\right] .
$$

ДокАЗАтЕльство. Так как $f, g$ лежат в $\mathfrak{T}^{+}$,то по предыдущей лемме $\widehat{f \mathscr{I}}, \widehat{\mathscr{I} g} \in \mathfrak{T}^{+}$, $\widehat{f \mathscr{I}} \geqslant \mathscr{I} \geqslant \widehat{\mathscr{I} g}$. А учитьвая 3) предыдущей леммы, убеждаемся, что классы $\widehat{\{f \mathscr{I}\}}$, $\{\widehat{\mathscr{I} g}\}$ можно считать замкнутыми относительно суперпозищии. Поэтому утверждение теоремы вытекает из теорем 1,2 .

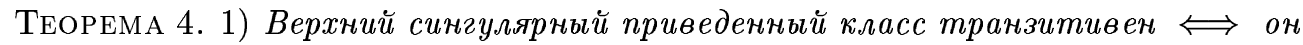
состоит из гибридной функиии вида $\widehat{h \mathscr{I}}$ для некоторой общерекурсивной $h$.

2) Если нижний транзитивный сингулярный приведенный класс состоит из функиии $e_{h}^{0}$, где $h-$ o.p.ф., то $e_{t \circ t}^{0}={ }_{\mathfrak{f}} e_{h}^{0}={ }_{\mathfrak{f}} e_{t}^{0}$, где $t=\min (h, \mathscr{I})$. Если для тотальной $h, h \unlhd \mathscr{I}$, выполняется условие, что $e_{h}^{0}$ является $I$-гибридной, то $\left\{e_{h}^{0}\right\}$ является сингулярным транзитивным нижним классом.

ДокАЗАтЕльство. 1) Сначала рассмотрим верхний класс $\{f\}$. Так как в этом случае речь идет о неубывающей о.р.ф., для которой дополнительно по следствию теоремы 2 выполняется условие $\mathscr{I} \unlhd f, f \circ f \unlhd f$, то по лемме 5,5$) f-\mathscr{I}$-гибридная.

2) Рассмотрим случай нижнего транзитивного класса. Предположим дан сингулярный приведенный транзитивный класс $\left\{e_{h}^{0}\right\}$. Имеем по условию рефлексивности $e_{h}^{0} \unlhd \mathscr{I}$. Теперь если $t=\min (h, \mathscr{I})$, то из $e_{h}^{0} \unlhd \mathscr{I}$ следует $e_{t}^{0}={ }_{\mathfrak{f}} e_{h}^{0}$. Действительно, на достаточно больших $x \in M_{h}$ значения $h, e_{h}^{0}$ совпадают и не превосходят $x$. А для таких $x$ и функция $t$ принимает те же значения, что и $h$. Ясно, что можно считать вьполненным дополнительно условие $e_{t}^{0} \in \mathfrak{T}^{+, \infty}$ (потому что если это неверно, то $e_{h}^{0}$ заключительно постоянна и гибридность $e_{h}^{0}$ вместе с утверждением теоремы очевидны).

Далее, из условия транзитивности имеем $e_{t}^{0} \unlhd e_{t \circ t}^{0}$. Пусть вновь $x-$ достаточно большое число из $M_{h}$. Из $t(x)=h(x)=e_{h}^{0}(x)=e_{t}^{0}(x)$ вытекает $t(t(x)) \leqslant e_{t}^{0}(x)$. Если бы последнее неравенство было строгим на бесконечно многих элементах $M_{h}$, то это давало бы противоречие с $e_{t}^{0} \unlhd e_{t \circ t}^{0}$. Отсюда почти всюду на $M_{h}\left(=M_{t}\right)$ функции $e_{t}^{0}$ и $e_{t \circ t}^{0}$ должны совпадать. Однако это и означает $e_{t}^{0}={ }_{f} e_{t \circ t}^{0}$.

Как известно, множество минимальньх элементов общерекурсивной функции иммунно или рекурсивно. В случае рекурсивности $e_{h}^{0}$ тоже рекурсивна. Но тогда по условию транзитивности должно быть $e_{h}^{0} \unlhd e_{h}^{0} \circ e_{h}^{0}$, учитывая еще, что $e_{h}^{0}$ не убывает. Теперь по лемме 5,5$) e_{h}^{0}$ является $\mathscr{I}$-гибридной. 
Достаточность условия, что $e_{h}^{0} \mathscr{I}$-гибридная, рассмотрена в теореме 3.

Некоторые функции могут составлять сингулярные классы, не будучи гибридньми. Например, тотальная мажоранта всех о.р.ф. составляет верхний транзитивный сингулярный класс. Аналогично тотальная миноранта $h \in \mathfrak{T}^{+, \infty}$ всех функций $e_{f}^{0} \in \mathfrak{T}^{+, \infty}$ для общерекурсивных $f$ составляет транзитивный нижний сингулярньй класс.

Перейдем к результатам, относяшимся к частичному упорядочению сводимостей $\leqslant_{m}\left[\begin{array}{l}\mathfrak{A}^{1} \\ \mathfrak{A}^{0}\end{array}\right]$ относительно включения. Обозначим это частично упорядоченное множество через $\mathfrak{M}$.

ТЕОРемА 5. Любой счетный частичный порядок изоморфно вкладывается в $\mathfrak{M}$.

ДОКАЗАТЕЛЬСТВО. Известно, что всякий частичньй порядок $(M, \leqslant l)$ изоморфно вкладывается в частично упорядоченное множество $\left(\left\{\mathfrak{S}_{i} \mid i \in M\right\}, \subset\right)$, где $\mathfrak{S}_{i}=$ $\{m \in M \mid m \leqslant l \quad i\}$. Пусть $\psi$ - взаимно однозначное отображение множества $M$ на множество всех простых чисел, больших 2. Определим $\mathfrak{S}_{i}^{\psi}=\bigcup_{p \in \psi\left(\mathfrak{S}_{i}\right)}\left\{p^{t} \mid t \geqslant 1\right\}$. Ясно, что $\left(M, \leqslant_{l}\right)$ изоморффно вкладьвается в $\left(\left\{\mathfrak{S}_{i}^{\psi} \mid i \in M\right\}, \subset\right)$. Теперь если $\mathfrak{S}_{i}^{\psi} \not \subset \mathfrak{S}_{j}^{\psi}$, то $\left|\mathfrak{S}_{i}^{\psi} \backslash \mathfrak{S}_{j}^{\psi}\right|=\infty$ и $\mathfrak{S}_{i}^{\psi} \backslash \mathfrak{S}_{j}^{\psi}$ включает бесконечное рекурсивное множество.

По каждому $\mathfrak{S}_{i}^{\psi}$ определим функцию $f_{i}: f_{i}(x)=x+C_{\mathfrak{S}_{i}^{\psi}}(x)$. Существенно, что для любых ненулевых $t, t^{\prime} \in \mathbb{N}$ и различных простых $p, p^{\prime} \in \psi(M)$ справедливо неравенство $\left|p^{t}-\left(p^{\prime}\right)^{t^{\prime}}\right| \geqslant 2$. Поэтому функции $f_{i}(x)$ являются $\mathscr{I}_{\text {-гибридными. }}$

Теперь положим $\mathfrak{R}_{i}^{1}=\left\{f_{i}\right\}$. Тогда отношения $\leqslant m\left[\begin{array}{c}\mathfrak{R}_{i}^{1} \\ \mathfrak{x}_{+}^{+}\end{array}\right]$определяют сводимости. Пусть $i, k \in M$ и $i<_{l} k$. Тогда $\mathfrak{S}_{i}^{\psi} \subset \mathfrak{S}_{k}^{\psi}$ и $\left|\mathfrak{S}_{k}^{\psi} \backslash \mathfrak{S}_{i}^{\psi}\right|=\infty$. Откуда $f_{i} \unlhd f_{k}$ и по лемме 2 $\leqslant_{m}\left[\begin{array}{c}\mathfrak{R}_{i}^{1} \\ \mathfrak{T}^{+}\end{array}\right] \subset \leqslant_{m}\left[\begin{array}{c}\mathfrak{R}_{k}^{1} \\ \mathfrak{T}^{+}\end{array}\right]$. Однако $f_{k}>f_{i}$ на некотором бесконечном рекурсивном множестве $\mathfrak{R}$ чисел $x$. По построению сушествует о.р.фp. $h \leqslant f_{k}$ такая, что $h(x)=x+C_{\mathfrak{R}}(x)$ и $h \not f_{i}$. Поэтому, используя лемму 4 , получаем $\leqslant m\left[\begin{array}{c}\mathfrak{R}_{i}^{1} \\ \mathfrak{T}^{+}\end{array}\right] \neq \leqslant m\left[\begin{array}{l}\mathfrak{R}_{k}^{1} \\ \mathfrak{T}^{+}\end{array}\right]$.

Если $i \|_{l} k$ в $\left(M, \leqslant_{l}\right)$, то $\left|\mathfrak{S}_{i}^{\psi} \backslash \mathfrak{S}_{k}^{\psi}\right|=\infty$ и $\left|\mathfrak{S}_{k}^{\psi} \backslash \mathfrak{S}_{i}^{\psi}\right|=\infty$, а поэтому $f_{i} \nsupseteq f_{k}$ и $f_{k} \nsupseteq f_{i}$. Это означает, что есть такие о.р.ф. $h_{i}, h_{k}$, что $h_{i} \leqslant f_{i}, h_{k} \leqslant f_{k}$ и $h_{i} \nsupseteq f_{k}$, а $h_{k} \nsupseteq f_{i}$. Следовательно, $\leqslant m\left[\begin{array}{l}\mathfrak{R}_{i}^{1} \\ \mathfrak{T}_{+}^{+}\end{array}\right]$и $\leqslant m\left[\begin{array}{c}\mathfrak{R}_{k}^{1} \\ \mathfrak{T}_{+}^{+}\end{array}\right]$несравнимы по включению. Это и доказывает, что построено изоморфное вложение $\left(M, \leqslant_{l}\right)$ в $(\mathfrak{M}, \subseteq)$.

Перейдем к результатам относительно частичных порядков степеней неразрешимости некоторых из построенных сводимостей. Мы отобрали утверждения, демонстрирующие существенные отличия стандартной $m$-сводимости от ряда построенных нами ограниченных сводимостей.

Обозначим через $d_{\mathfrak{R}^{0}}^{\Re^{1}}(A)$ степень неразрешимости множества $A$ относительно сводимости $\leqslant m\left[\begin{array}{l}\mathfrak{R}^{1} \\ \mathfrak{R}^{0}\end{array}\right]$. Стандартно определяемое частично упорядоченное множество $\left(\left\{d_{\mathfrak{R}^{0}}^{\mathfrak{R}^{1}}(A) \mid A \subseteq \mathbb{N}\right\}, \leqslant\right)$ будем обозначать через $L_{\mathfrak{R}^{0}}^{\mathfrak{R}^{1}}$.

С ледующая теорема показывает, что взаимодействие верхних и нижних ограничений может порождать разветвленную систему степеней даже в классе рекурсивных множеств.

ТЕОРемА 6. Пусть $\mathfrak{P}$ обозначает класс одноместных полиномов с натуральными коэффиииентами. Тогда $L_{\{\mathscr{I}\}}^{\mathfrak{P}}$ включает бесконечные убывающие и возрастаюшие иепи рекурсивных степеней и бесконечные совокупности попарно несравнимых рекурсивных степеней. 
ДоКАЗАТЕЛЬСТВо. Заметим, что используемые классы, очевидно, образуют сводимость. Для доказательства построим конкретные множества. Пусть $A=\{y(i) \mid$ $\left.y(0)=0, y(1)=2, y(i+1)=2^{y(i)}, i \in \mathbb{N}\right\}$.

Теперь рассмотрим множества $B_{i}=\left\{x \mid x=p_{i}^{j}, j \in \mathbb{N}\right\}$, где $p_{i}-i$-е простое число. Это бесконечные рекурсивные множества. Пусть $C_{0}=A=\{y(i) \mid i \in \mathbb{N}\}$, a $C_{n+1}=C_{n} \backslash\left\{y(k) \mid k \in B_{n+1}\right\}$, т.е. каждое следующее множество мы получаем, выбрасывая из предыдущего бесконечное рекурсивное подмножество. Далее положим $D_{0}=\left\{y(k) \mid k \in B_{1}\right\}, D_{n+1}=D_{n} \cup\left\{y(k) \mid k \in B_{n+2}\right\}$. (Здесь на каждом шаге добавляем бесконечное рекурсивное множество.) Ясно, что $\mathrm{C}_{n+1} \leqslant g_{m}^{g_{n}}\left[\begin{array}{l}\mathfrak{P} \\ \{\mathscr{I}\}\end{array}\right] C_{n}$, где

$$
g_{n}(x)= \begin{cases}x+1, & \text { если } x \in C_{n} \backslash C_{n+1}, \\ x & \text { иначе. }\end{cases}
$$

Докажем, что $C_{n} \Varangle_{m}\left[\begin{array}{l}\mathfrak{P} \\ \{\mathscr{I}\}\end{array}\right] C_{n+1}$. Действительно, в противном случае существует $k \in \mathbb{N}$ и $f(x)$ - сводящая функция такая, что $\mathscr{I}(x) \unlhd f(x) \unlhd x^{k}$. Найдется такое $x_{0} \in \mathbb{N}$, зависяшее от $k$, что $\forall x: x>x_{0}\left[y(x),(y(x))^{k}\right] \subset[y(x), y(x+1)]$. Тогда образ любого элемента из $\left\{y(k) \mid k \in B_{n+1}, k>x_{0}\right\}$ не может принадлежать $C_{n+1}$. А это противоречит сводимости.

Совершенно аналогично $D_{n} \leqslant m\left[\begin{array}{l}\mathfrak{P} \\ \{\mathscr{I}\}\end{array}\right] D_{n+1}$ и $D_{n+1} \Varangle_{m}\left[\begin{array}{l}\mathfrak{P} \\ \{\mathscr{I}\}\end{array}\right] D_{n}$.

Рассмотрим вторую часть утверждения теоремы. Пусть $F_{n}=\left\{y(i) \mid i=2^{n} \cdot q, q-\right.$ нечетное $\}$. Тогда если $k \neq j$, то, как и раньше, $F_{k} \Varangle_{m}\left[\begin{array}{l}\mathfrak{P} \\ \{\mathscr{I}\}\end{array}\right] F_{j}$ и $F_{j} \nless_{m}\left[\begin{array}{l}\mathfrak{P} \\ \{\mathscr{I}\}\end{array}\right] F_{k}$.

Достаточно низкие верхние гранишы могут привести к такой патологии, как отсутствие точной верхней грани для системы соответствующих степеней.

TЕорема 7. Пусть $\leqslant m\left[\begin{array}{l}\mathfrak{R}^{1} \\ \mathfrak{R}^{0}\end{array}\right]-$ сводимость. Если $\forall c \in \mathbb{N}$ в $\mathfrak{R}^{1}$ нет функиии, мажсорирующей п.в. функиию $2 \mathscr{I}-c$, то $L_{\mathfrak{R}^{0}}^{\mathfrak{R}^{1}}$ не является верхней полурешеткой.

ДокАЗАТЕльство. Для доказательства будем использовать понятие $\mathfrak{T}^{+}-$несжимаемого множества, рассмотренного в [4]. Возьмем рекурсивно несжимаемое множество $A$ и рассмотрим его как сочленение $B \oplus C$. Тогда $B=\{a \mid 2 a \in A\}, C=\{a \mid$ $2 a+1 \in A\}$. Они тоже рекурсивно несжимаемы в силу леммы 2 из [4]. Пусть $S$ - наименьшая верхняя грань в $L_{\mathfrak{R}^{0}}^{\mathfrak{R}^{1}}$ для $B$ и $C$. По условию для любого $c \in \mathbb{N}$ множество $A$ не может быть сведено данной сводимостью к $S$ с границей, мажорирующей $2 \mathscr{I}-c$. Поэтому $A \leqslant \mathfrak{R}_{\mathfrak{R}^{0}}^{1} S$ с верхней границей из $\mathfrak{T}^{+}$, почти всюду мажорируемой $2 \mathscr{I}-t_{A}$, где $t_{A} \in \mathfrak{T}^{+, \infty}$. То же верно и для множества $B$ и некоторой $t_{B} \in \mathfrak{T}^{+, \infty}$.

Но тогда $A \leqslant(\mathscr{I} \dot{-} h) S$, где $h \in \mathfrak{T}^{+, \infty}$, и следовательно, $A$ рекурсивно сжимаемо. Это противоречие и доказьвает теорему.

СлЕДСТВИЕ 3. Для любой сводимости типа $\leqslant_{m}\left[\begin{array}{l}\widehat{f \mathscr{I}} \\ {\left[\mathfrak{R}^{0}\right.}\end{array}\right]$ система ее степеней неразрешимости не является верхней полурешеткой.

ДоКАЗАТЕЛЬСТВО непосредственно следует из теоремы 7.

В заключение коснемся темы полноты для вновь введенных сводимостей. Как видно из дальнейшего, здесь также ситуация осложняется.

Пусть $\leqslant_{m}\left[\begin{array}{l}\mathfrak{R}^{1} \\ \mathfrak{R}^{0}\end{array}\right]-$ сводимость. По аналогии с классическими сводимостями будем говорить, что множество $A$ полно относительно $\leqslant m\left[\begin{array}{l}\mathfrak{R}^{1} \\ \mathfrak{R}^{0}\end{array}\right]$, если

1) $A$-р.п.м.;

2) $(\forall B)\left[B-\right.$ р.п.м. $\Longrightarrow B \leqslant m\left[\left[_{\mathfrak{R}^{0}}^{\mathfrak{R}^{1}}\right] A\right]$. 
Например, рассмотрим множество $K_{0}=\left\{\langle x, y\rangle \mid x \in W_{y}\right\}$. Ясно, что если р.п.м. $B=W_{y_{0}}$, то $B \leqslant m,\left\langle x, y_{0}\right\rangle K_{0}$. Теперь если зафиксировать некоторую нумерацию, например, $\langle x, y\rangle=\frac{1}{2}\left((x+y)^{2}+3 x+y\right)$, то получим, что $K_{0}$ является $\leqslant m\left[\begin{array}{l}\mathfrak{P} \\ \mathfrak{R}^{0}\end{array}\right]$-полньц, если $\mathfrak{P}-$ класс всех одноместных полиномов с натуральными коэффициентами. Это так, потому что $(\exists p \in \mathfrak{P})(\forall z)[p(x) \unrhd\langle x, z\rangle]$.

Введем теперь следующее определение. Совокупность множеств $\mathfrak{K}\left(\mathfrak{K} \subset 2^{\mathbb{N}}\right)$ будем назьвать полной системой множеств для сводимости $\leqslant m\left[\begin{array}{l}\mathfrak{R}^{1} \\ \mathfrak{R}^{0}\end{array}\right]$, если

1) $(\forall A \in \mathfrak{K})[A-$ р.п.м.];

2) $(\forall$ р.п.м. $B) \exists A\left[A \in \mathfrak{K} \& B \leqslant m\left[\begin{array}{l}\mathfrak{R}^{1} \\ \mathfrak{R}^{0}\end{array}\right] A\right]$.

Полные системы множеств (далее п.с.м.) всегда существуют. Действительно, множество всех р.п.м. образует п.с.м. Для классической $\leqslant m$-сводимости п.с.м. состоит из одного множества - множества $K$.

П.с.м. для данной сводимости назовем нетривиальной, если она не включает никакого полного для данной сводимости множества.

Обозначим через $\mathfrak{I}^{+}$класс $\{\mathscr{I}+c \mid c \in \mathbb{N}\}$.

ТЕорема 8. Для сводимости $\leqslant\left[\mathfrak{\Im}_{\mathfrak{T}^{+}}^{+}\right]$не существует полных мноэкеств. (Это означает, что все п.с.м. для $\leqslant\left[\mathfrak{\mathfrak { T }}^{+}\right]$нетривиальны.)

ДокАЗАТЕЛЬСтво. Пусть, напротив, р.п.м. $A \leqslant\left[\mathfrak{\Im}_{\mathfrak{T}}^{+}\right]-$полно. Ввиду рекурсивной перечислимости $A$ оно рекурсивно сжимаемо (см. [4]), т.е. $\exists A^{\prime}, A^{\prime}$ р.п.м., $A^{\prime} \leqslant_{T} A$ и $A \leqslant \mathscr{I} \dot{ }_{h} A^{\prime}$ для некоторой $h \in \mathfrak{T}^{+, \infty}$.

Теперь если $A^{\prime} \leqslant\left[\left[_{\mathfrak{I}^{+}}^{\mathfrak{I}^{+}}\right] A\right.$, то $\exists c \in \mathbb{N}$ такое, что $A^{\prime} \leqslant \stackrel{\mathscr{I}}{m}^{+c} A$. Но из $A \leqslant \mathscr{I} \dot{h}_{h} A^{\prime}$ по транзитивности $\leqslant T$ вытекает, что $A \leqslant(\mathscr{I} \dot{\dot{h}} h)+c A$. Это означает, однако, что $A \leqslant \mathscr{I} \dot{-} h^{\prime} A$, где $h^{\prime}=h-c \& h^{\prime} \in \mathfrak{T}^{+, \infty}$. Это в свою очередь влечет рекурсивность $A$. Но рекурсивное множество вообще не может быть полным.

\section{СПИСОК ЦИТИРОВАННОЙ ЛИТЕРАТУРЫ}

[1] Булитко В. К. Субтьюринговы сводимости ограниченной сложности // Изв. вузов. Матем. 1992. T. 1. C. 7-17.

[2] Булитко B. K. About Segment Complexity of Turing Reductions // Math. Log. Quart. 1999. T. 45. № 4. C. 561-571.

[3] Роджерс Х. Теория рекурсивных функций и эффективная вычислимость. М.: Мир, 1972.

[4] Булитко В. К. О рекурсивно сжимаемых множествах // Матем. заметки. 1998. Т. 64. № 1. C. 9-16. 